\title{
PULSED SURFACE HEATING OF A SEMI-INFINITE SOLID*
}

By J. C. JAEGER (Australian National University)

1. Introduction. In many practical systems heat is supplied to the surface of a solid in regular rectangular pulses, so that the flux of heat $F$ at the surface at time $t$ will be

$$
\left.\begin{array}{l}
F=F_{0}, \quad n T<t<n T+T_{1} \\
F=0, \quad n T+T_{1}<t<(n+1) T,
\end{array}\right\} n=0,1,2, \cdots
$$

where $F_{0}, T$, and $T_{1}$ are constants and the solid is supposed to be at zero temperature when $t=0$. Such problems occur, for example, in "on-off" heating, in heat generation by friction over portion of the surface of a rotating cylinder ${ }^{1}$, in the rotating anode $\mathrm{X}$-ray tube ${ }^{2}$, in the heating of machine guns, in the heating of the anode of a magnetron, and so on.

It is usually desired to know the surface temperature after steady conditions have been attained, and, in particular, the temperature at the end of a heating interval. Fourier series are not very suitable for the treatment of such problems (since their convergence is very slow at the most interesting values of the time) and alternative methods have been given by Weber ${ }^{3}$ and Oosterkamp ${ }^{2}$, but the most powerful and widely applicable method seems to be that of the Laplace transformation ${ }^{4}$ in a form which is essentially equivalent to the steady-state operational calculus of Waidelich ${ }^{5}$.

To illustrate the method the most important case, that of the semi-infinite solid heated over the whole of its surface, is discussed in some detail in \$2: the results of this section are frequently useful as approximations for the case of a finite solid similarly heated.

The more difficult problem of the semi-infinite solid with heat supplied over a circular area of its surface is of interest in connection with rotating anode X-ray tubes and is discussed in §3. Results for pulsed point and line sources in infinite medium are given in $\$ 4$.

2. The semi-infinite solid heated over the whole of its surface.

The semi-infinite solid $x>0$, of conductivity $k$ and diffusivity $\alpha$, initially at zero temperature, is heated over the whole of the surface $x=0$ by the flux (1). It is required to find its surface temperature for large values of the time.

If $v$ is the temperature in the solid we have to solve

$$
\frac{\partial^{2} v}{\partial x^{2}}-\frac{1}{\alpha} \frac{\partial v}{\partial t}=0, \quad x>0, \quad t>0
$$

with

$$
\begin{gathered}
v=0, \text { when } t=0, x>0, \\
-k \frac{\partial v}{\partial x}=F, \quad x=0, \quad t>0,
\end{gathered}
$$

\footnotetext{
*Received May 5, 1952.

1Jaeger, Phil. Mag., (7), 35, 169 (1944).

2Oosterkamp, Philips Res. Rep., 3, 161 (1948).

${ }^{3}$ Weber, Ann. der Phys., 146, 257 (1872).

${ }^{4}$ Carslaw and Jaeger, Operational Methods in Applied Mathematics, Ed. 2 (1948), §129.

${ }^{6}$ Waidelich, Proc. Inst. Radio Engrs., 23, 78P (1946).
} 
and $v$ finite as $x \rightarrow \infty$, where $F$ is given by (1). Writing

$$
v^{*}=\int_{0}^{\infty} e^{-s t} v d t
$$

for the Laplace transform of $v$, with a similar notation for that of $F$, etc., we have from (1)

$$
F^{*}=\frac{F_{0}\left(1-e^{-s T_{1}}\right)}{s\left(1-e^{-s T}\right)}
$$

and, by the usual Laplace transformation procedure ${ }^{4}$

$$
v^{*}=\frac{F_{0} \alpha^{1 / 2}\left(1-e^{-s T_{1}}\right) e^{-x s^{1 / 2} \alpha^{1 / 2}}}{k s^{3 / 2}\left(1-e^{-s T}\right)} .
$$

This gives for the surface temperature $v_{0}$ at $x=0$,

$$
\begin{array}{ll}
v_{0}=\frac{2 F_{0}}{k}\left(\frac{\alpha t}{\pi}\right)^{1 / 2}, & 0<t<T_{1}, \\
v_{0}=\frac{2 F_{0} \alpha^{1 / 2}}{k \pi^{1 / 2}}\left\{t^{1 / 2}-\left(t-T_{1}\right)^{1 / 2}\right\}, & T_{1}<t<T,
\end{array}
$$

and so on. Also, the inversion theorem for the Laplace transformation gives

$$
v_{0}=\frac{F_{0} \alpha^{1 / 2}}{2 \pi i k T} \int_{\gamma-i \infty}^{\gamma+i \infty}\left\{\frac{T-T e^{-s T_{2}}-T_{1}+T_{1} e^{-s T}}{1-e^{-s T}}+T_{1}\right\} e^{s t} s^{-3 / 2} d s,
$$

for all values of $t$, where $\gamma$ is a positive constant. The integrand of (10) has a branch point at $s=0$ and simple poles at

$$
s= \pm 2 n \pi i / T, \quad n=1,2, \cdots .
$$

When the integral (10) is evaluated by contour integration in the usual way, the residues at these poles give rise to a series of terms with period $T$, and the sum of this series, which we shall denote by $v_{P}$, is the required steady periodic term. The final result is

$$
v_{0}=\frac{2 F_{0} T_{1}}{k T}\left(\frac{\alpha t}{\pi}\right)^{1 / 2}+v_{P}-\frac{2 F_{0} \alpha^{1 / 2}}{\pi k T} \int_{0}^{\infty} \frac{e^{-u^{3} t}\left(T-T e^{u^{3} T_{1}}-T_{1}+T_{1} e^{u^{3} T}\right) d u}{u^{2}\left(1-e^{u^{2} T}\right)},
$$

where the first term of (12) comes from the last term in the brace in (10), and the integral in (12) comes from the branch point in the first term in the brace in (10).

For large values of the time the integral in (12) is negligible, and $v_{0}$ is given by the steady periodic part $v_{P}$ superposed on the first term of (12) which corresponds to steady heating by the average flux $F_{0} T_{1} / T$ commencing at $t=0$.

For small values of $t$ the result (12) must agree with (8) for $0<t<T_{1}$, and with (9) for $T_{1}<t<T$, and this gives an integral expression for $v_{P}$ over the whole of a period. Writing

$$
a=T_{1} / T, \quad Q=F_{0} T_{1},
$$


the value of $v_{P}$ at time $b T$ after the beginning of a period is

$$
\begin{array}{ll}
v_{P}=\frac{2 Q}{k a}\left(\frac{\alpha}{\pi T}\right)^{1 / 2}\left\{(1-a) b^{1 / 2}-\pi^{-1 / 2} I(a, b)\right\}, & 0<b<a \\
v_{P}=\frac{2 Q}{k a}\left(\frac{\alpha}{\pi T}\right)^{1 / 2}\left\{(1-a) b^{1 / 2}-(b-a)^{1 / 2}-\pi^{-1 / 2} I(a, b)\right\}, & a<b<1,
\end{array}
$$

where

$$
I(a, b)=\int_{0}^{\infty} \frac{e^{-b \xi^{2}}\left\{(1-a) e^{-\xi^{2}}-e^{-(1-a) \xi^{2}}+a\right\} d \xi}{\xi^{2}\left(1-e^{-\xi^{2}}\right)} .
$$

These integrals are easy to evaluate numerically. The values of $a$, the ratio of the heating time $T_{1}$ to the period $T$, vary greatly in practice. For on-off switching and frictional heating of a rotating cylinder they may be relatively large, but in other systems, such as machine guns or the anodes of X-ray tubes, they are usually of the order of 0.02 or less. Values of $v_{P}$ at time of $b T$ after the beginning of a period are shown in Curves I and II of Fig. 1 for $a=0.5$ and 0.1 , respectively, for the same quantity of heat $Q$ emitted per unit area per cycle.

Curve III of Fig. 1 corresponds to the case $a \rightarrow 0$, that is, to the quantity of heat $Q$ per unit area being supplied instantaneously at the beginning of each period so that (1) is replaced by

$$
F=Q \sum_{n=0}^{\infty} \delta(t-n T),
$$

where $\delta(t)$ is the Dirac delta function.

In this case (14) and (15) are replaced by

$$
v_{P}=\frac{Q}{k}\left(\frac{\alpha}{\pi T}\right)^{1 / 2}\left\{b^{-1 / 2}-2 b^{1 / 2}-\frac{2}{\pi^{1 / 2}} \int_{0}^{\infty} \frac{e^{-b \xi^{2}}\left\{1-\left(\xi^{2}+1\right) e^{-\xi^{2}}\right\} d \xi}{\xi^{2}\left(1-e^{-\xi^{2}}\right)}\right\} .
$$

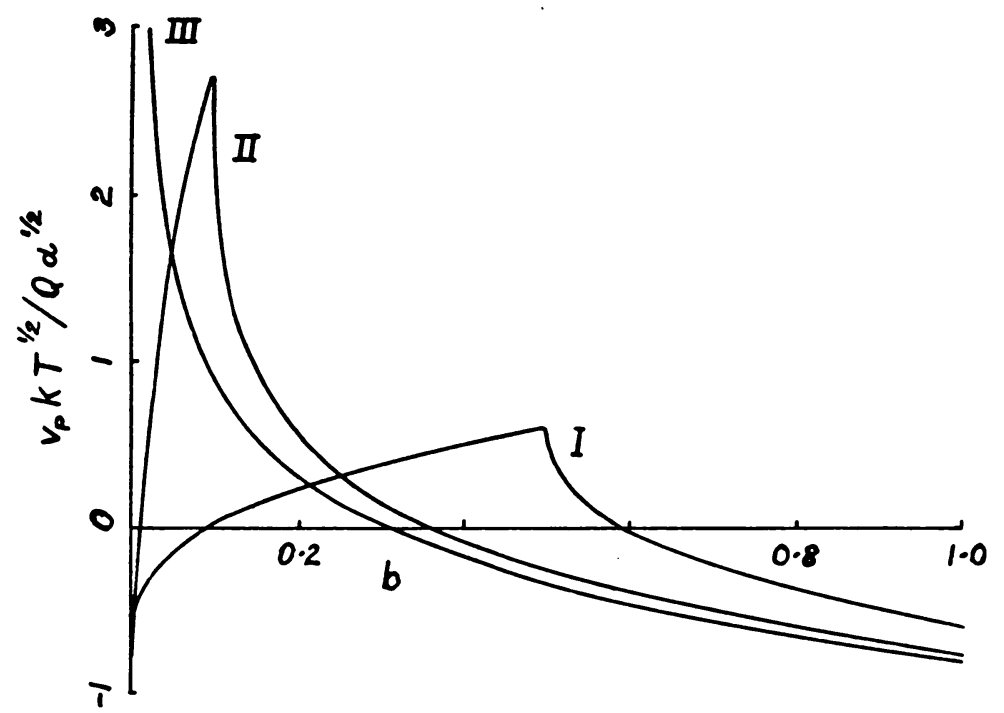

FIG. 1. Periodic temperature ascillations of the surface of a semi-infinite solid when the same quantity of heat is liberated over half the cycle (Curve I), over 1/10 of the cycle (Curve II), and instantaneously (Curve III). 
The most important quantities in practice are the temperatures at the beginning and end of a heating interval, obtained by taking $b=0$ and $b=a$, respectively in (14). The variation of these with the parameter $a=T_{1} / T$ for the same total quantity of heat $Q$ supplied per unit area per cycle is shown in Fig. 2, Curves I and II.

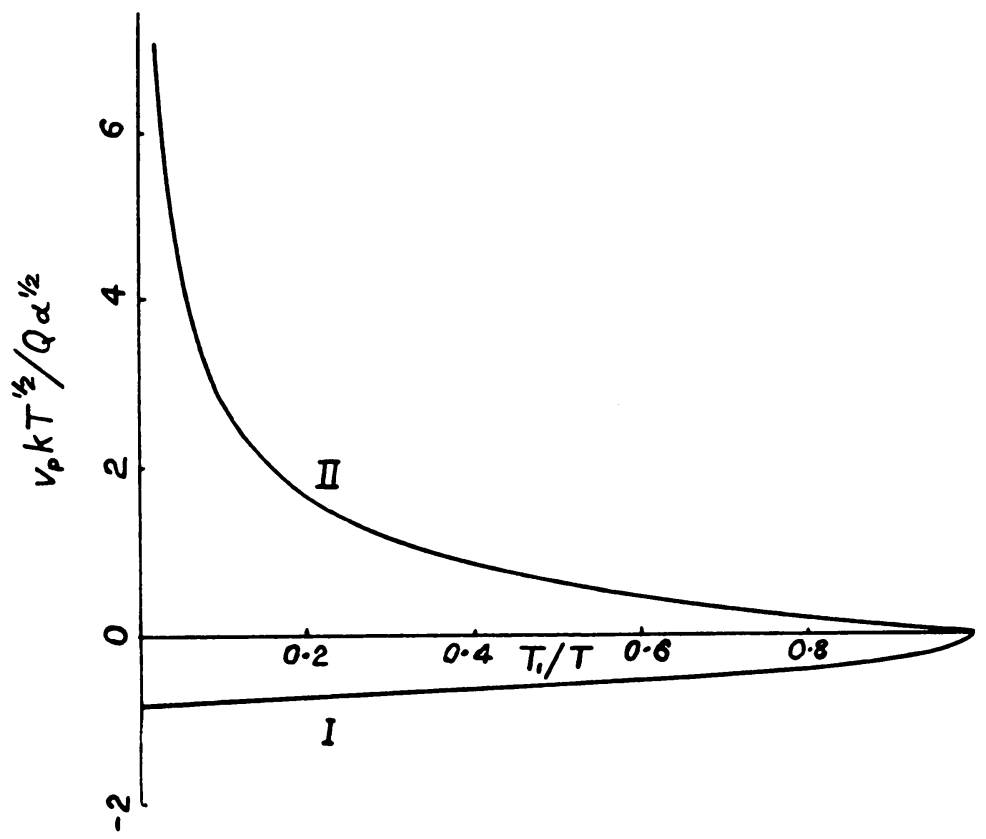

Fig. 2. Periodic Temperature at the beginning (Curve I) and end (Curve II) of a heating interval.

Finally it may be remarked that there is no difficulty in writing down expressions for the temperature at depth $x$. For example, for the surface flux (17) the temperature at depth $x$ at a time $t$ after the beginning of heating which is so long that the starting transient has disappeared is

$$
\frac{2 Q(\alpha t)^{1 / 2}}{k T} \operatorname{ierfc} \frac{x}{2(\alpha t)^{1 / 2}}+v_{P},
$$

where the value of the periodic part at time $b T$ after the beginning of a heating interval is

$$
\begin{aligned}
v_{P}=\frac{Q \alpha^{1 / 2}}{k(\pi b T)^{1 / 2}} e^{-c^{2 / 4} / 4}-\frac{2 Q \alpha^{1 / 2} b^{1 / 2}}{k T^{1 / 2}} & \operatorname{ierfc} \frac{c}{2 b^{1 / 2}} \\
& -\frac{2 Q \alpha^{1 / 2}}{\pi k T^{1 / 2}} \int_{0}^{\infty} \frac{e^{-b \xi^{2}}\left[1-\left(1+\xi^{2}\right) e^{-\xi^{2}}\right] \cos c \xi}{\xi^{2}\left(1-e^{-\xi^{2}}\right)} d \xi,
\end{aligned}
$$

where

$$
\operatorname{ierfc} x=\int_{x}^{\infty} \operatorname{erfc} \xi d \xi,
$$

$c=x(\alpha T)^{-\frac{1}{2}}$, and $a$ and $Q$ are defined in (13).

It follows from (19) that, for large values of the time $t$, the temperature is accurately the sum of the periodic oscillation $v_{P}$ whose mean value is zero and a term corresponding to heating for time $t$ by the constant flux $Q / T$, the mean value of the applied flux. The 
same remark applies to all the other cases discussed. There seems to be no justification for the suggestion of Comenetz ${ }^{6}$ that the temperature behaves on the average as if the constant flux $Q / T$ had been supplied for time $t+\frac{1}{2} T$.

\section{The semi-infinite solid heated over a circular area.}

Suppose the semi-infinite solid is heated by flux (1) applied over a circle of radius $R$ in its surface, there being no loss of heat from the remainder of the surface. When steady conditions have been attained, the temperature at the centre of the circle at time $b T$ after the beginning of a period is found to be

$$
\frac{R Q}{k T}+v_{P}
$$

where, now, the steady periodic part $v_{P}$ is given by

$$
\begin{aligned}
v_{P}=\frac{Q \alpha^{1 / 2}}{k T^{1 / 2}}\left\{\frac{2 b^{1 / 2}}{a \pi^{1 / 2}}\left(1-e^{-c^{2 / 4 b}}\right)+\frac{c}{a} \operatorname{erfc} \frac{c}{2 b^{1 / 2}}-c\right. & \\
& +\frac{2}{\pi a} \int_{0}^{\infty} \frac{e^{-b \xi^{2}}\left\{e^{-(1-a) \xi^{2}}-e^{-\xi^{2}}\right\}\{1-\cos c \xi\} d \xi}{\xi^{2}\left(1-e^{-\xi^{2}}\right)}, \quad 0<b<a,
\end{aligned}
$$

where

$$
Q=F_{0} T_{1}, \quad a=T_{1} / T, \quad c=R /(\alpha T)^{1 / 2} .
$$

As before, the most interesting quantity is the maximum value of $v_{P}$ which is attained when $b=a$. Values of this, plotted against $T_{1} / T$, are shown in Fig. 3 for various values of the parameter $c$. For $c<0.2$ the integrals in (22) do not make an important contribution, while for $c=1$ they are already close to the limiting case $c \rightarrow \infty$ of Fig. 2, Curve II.

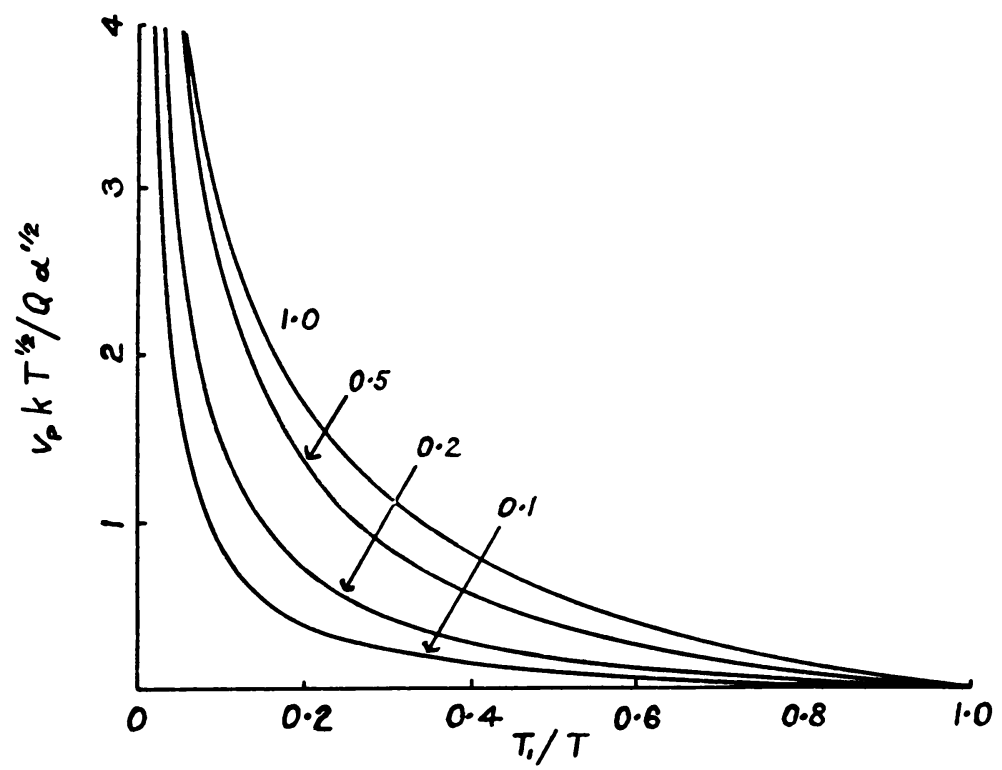

FIG. 3. Values of the periodic temperature at the end of a heating interval for heat supply over a circle of radius $R$. The numbers on the curves are the values of $R(\alpha T)^{-\frac{1}{3}}$.

'Quart. App. Math., 5, 503 (194י). 


\section{Pulsed point and line sources in an infinite medium.}

For the point source, suppose that heat is supplied at the origin at the rate of $q$ units per unit time in the time intervals

$$
n T<t<n T+T_{1}, \quad n=0,1,2, \cdots
$$

no heat being supplied at other times. Then, when steady conditions have been attained, the temperature at distance $r$ from the origin at a time $b T$ after the beginning of a heating period is, for $0<b<a$,

$$
\frac{q a}{4 \pi k r}+v_{P}
$$

where the periodic part $v_{P}$ is

$$
v_{P}=\frac{q}{4 \pi k r}\left\{\operatorname{erfc} \frac{C}{2 b^{3 / 2}}-a+\frac{2}{\pi} \int_{0}^{\infty} \frac{e^{-b \xi^{2}}\left[e^{-(1-a) \xi^{2}}-e^{-\xi^{2}}\right] \sin C \xi}{\xi\left(1-e^{-\xi^{2}}\right)} d \xi\right\},
$$

and

$$
a=T_{1} / T, \quad C=r(\alpha T)^{-1 / 2} .
$$

This is the limiting case of the problem of $\$ 3$.

For the line source, suppose that heat is supplied along an infinite line at the rate $q$ units per unit length per unit time during the time intervals (24), no heat being supplied at other times. Then, for large values of the time the temperature at distance $r$ from the line is, for $0<b<a$,

$$
-\frac{q a}{4 \pi k} E i\left(-\frac{r^{2}}{4 \alpha t}\right)+v_{P}
$$

where the periodic part $v_{P}$ is

$v_{P}=-\frac{q(1-a)}{4 \pi k} E i\left(-\frac{C^{2}}{4 b}\right)-\frac{q}{2 \pi k} \int_{0}^{\infty} \frac{e^{-b \xi^{2}} \cdot J_{0}(C \xi)\left[(1-a) e^{-\xi^{2}}-e^{-(1-a) \xi^{2}}+a\right] d \xi}{\xi\left(1-e^{-\xi^{2}}\right)}$,

and $a$ and $C$ are defined in (27), $E i$ is the exponential integral, and $J_{0}$ the Bessel function of the first kind of order zero.

\section{BOOK REVIEWS}

Tensor analysis for physicists. By J. A. Schouten. The Clarendon Press, Oxford, 1951. $x+275$ pp. 50 s.

The first five chapters of this extremely well written book are devoted to an exposition of tensor analysis and Riemannian geometry. The author uses his well known expository skill to give a clear geometric interpretation to the various quantities discussed. The last five chapters are devoted to applications of this material to various physical problems. The two groups of chapters are separated by a summary of the salient points of the theory described in the first part of the book. This section should prove to be very useful.

Chapters I and II deal with the algebra of affinors (tensors) and contain a detailed discussion of $p$-vectors and $p$-vector densities (anti-symmetric tensors and tensor densities with $p$ indices). The !dentification of various affinors obtained by restricting the group of the space is made extremely clear. 